\title{
Outer Plexiform Layer Angle: A Prognostic Factor for Idiopathic Macular Pucker Surgery
}

\author{
Ding-Ying Liao, ${ }^{1}$ Jorn-Hon Liu, ${ }^{2}$ Yu-Ping Zheng, ${ }^{1}$ Jian-Ming Wang $\mathbb{D},{ }^{1}$ \\ and Hsiao-Ming Chao $\mathbb{D D}^{3,4,5}$ \\ ${ }^{1}$ Department of Ophthalmology, Second Affiliated Hospital, Xi'an Jiaotong University, Xi'an, Shaanxi, China \\ ${ }^{2}$ Department of Ophthalmology, Cheng Hsin General Hospital, Taipei, Taiwan \\ ${ }^{3}$ Institute of Pharmacology, School of Medicine, National Yang-Ming University, Taipei, Taiwan \\ ${ }^{4}$ Department of Chinese Medicine, School of Chinese Medicine, China Medical University, Taichung, Taiwan \\ ${ }^{5}$ Department of Ophthalmology, Shin Kong Wu Ho-Su Memorial Hospital, No. 95 Wenchang Rd, Shilin District, Taipei, Taiwan
}

Correspondence should be addressed to Jian-Ming Wang; xajdwjm@163.com and Hsiao-Ming Chao; hsiaoming.chao@gmail.com

Received 12 August 2020; Revised 17 October 2020; Accepted 28 October 2020; Published 16 November 2020

Academic Editor: Dirk Sandner

Copyright (c) 2020 Ding-Ying Liao et al. This is an open access article distributed under the Creative Commons Attribution License, which permits unrestricted use, distribution, and reproduction in any medium, provided the original work is properly cited.

\begin{abstract}
Purpose. To investigate the efficacy of idiopathic macular pucker (epimacular membrane) surgery and to identify the possible prognostic factor. Methods. This was a retrospective study which enrolled 38 patients with idiopathic macular pucker who underwent 23-gauge pars plana vitrectomy (PPV) with indocyanine green-assisted peeling of epiretinal membrane (ERM) and internal limiting membrane (ILM). Visual outcomes were assessed at the baseline and during the follow-up including bestcorrected visual acuity (BCVA) and metamorphopsia score, as well as outer plexiform layer (OPL) angle and central retinal thickness (CRT) using spectral-domain optical coherence tomography (SD-OCT). A comparison was made between patients with the prepeeling CRTs $\geq 500 \mu \mathrm{m}$ and those $<500 \mu \mathrm{m}$. A comparison was also made between patients with the prepeeling OPL angles $\geq 130^{\circ}$ and those $<130^{\circ}$. Based on the prepeeling parameters, the correlations between various prepeeling and postpeeling visual functions were analyzed. Results. Mean follow-up was 36.07 \pm 4.62 months (range 1.30-96.70 months). BCVA was significantly improved from $0.26 \pm 0.03$ to $0.67 \pm 0.04(p<0.001)$; metamorphopsia score was significantly reduced from $1.42 \pm 0.16$ to $0.61 \pm 0.08$ ( $p<0.001)$; CRT was significantly decreased from $519.62 \pm 13.41 \mu \mathrm{m}$ to $385.37 \pm 8.97 \mu \mathrm{m}(p<0.001)$. Greater prepeeling OPL angle $\left(\geq 130^{\circ}\right)$ was associated with significantly greater BCVA improvement (Snellen E/LogMAR: $\left.p=0.01 / 0.03\right)$ and greater metamorphopsia reduction $(p=0.03)$, as compared to smaller OPL angle $\left(<130^{\circ}\right)$ with less BCVA improvement and less metamorphopsia reduction. However, the final BCVA improvement and metamorphopsia reduction relevant to the prepeeling smaller CRT $(<500 \mu \mathrm{m})$ did not significantly differ from that relevant to the prepeeling greater CRT $(\geq 500 \mu \mathrm{m} ; p>0.05)$. Endophthalmitis, retinal tear, or retinal detachment was not observed after peeling. Conclusion. Indocyanine green-assisted ERM/ ILM peeling combined with small gauge vitrectomy is associated with significant visual acuity improvement and metamorphopsia reduction in patients with idiopathic macular pucker. Greater prepeeling OPL angle rather than CRT might act as a useful prognostic factor in predicting better final visual functional outcomes.
\end{abstract}

\section{Introduction}

Idiopathic macular pucker or epiretinal membrane (ERM) is a common disease, which is associated with fibrocellular proliferative tissue growing along the vitreoretinal interface in the macular area. The prevalence of this disease is reported to be $2 \%$ in patients under the age of 60 years and $12 \%$ in those over the age 70 years [1]. In the early stage of macular pucker, patients generally do not present with obvious symptoms. As the disease progresses, it is said to cause a number of visual function and retinal anatomy alterations, such as decreased best-corrected visual acuity (BCVA), metamorphopsia, increased central retinal thickness (CRT), retinal wrinkling, and visual distortion [1-7]. The symptoms 
listed above (e.g., metamorphopsia) can lead to vision impairment and thus have detrimental effects on their day-today activities [1-7]. In this case, the spectrum-domain optical coherence tomography (SD-OCT) is a widely known and useful tool for the diagnosis and classification of macular pucker, via providing high-resolution cross-sectional scans of various retinal layers and microstructures.

Surgical correction is usually recommended as a standard procedure for improving visual outcomes via releasing tractional force of the epiretinal membrane [2]. However, some patients still report with poor visual outcomes, despite successful operative procedures [3]. A comparison has been made between preoperative prognostic factors and postoperative ones such as duration of blurred vision [4], visual acuity [3], status of inner segment/outer segment (IS/OS) junction, cone outer segment tips (COSTs), and photoreceptor outer segment (PROS) length [5-7]. On the other hand, pars plana vitrectomy (PPVT) together with removal of ERM/internal limiting membrane (ILM) is an effective and useful treatment; however, the rates of the ERM recurrence and the reoperation are in $10 \%$ and $3 \%$, respectively [8]. In this case, ILM peeling that removes the scaffold for myofibroblast proliferation along with the ERM removal might minimize the recurrence of ERM [9]. Of note, ILM itself is a layer of transparent tissue which thus makes it hard to be visualized during the operation. Thus, indocyanine green is presently injected to stain the ILM as an attempt to enhance its visualization. PPVT together with ICG-assisted ILM peeling and ERM removal has demonstrated satisfactory anatomic and visual outcomes in many patients; however, some others still showed poor visual outcomes [10].

Therefore, it is important to conduct a retrospective medical record review of patients who underwent PPVT with ICG-assisted ILM peeling and EMM removal to investigate the efficacy of the surgery. The present evaluations also included prepeeling prognostic factors, for example, the preoperative outer plexiform layer (OPL) angle and CRT that are related to postoperative BCVA and metamorphopsia score. OPL angle might be a significantly and clinically important factor that could be helpful in predicting postpeeling visual outcomes and justify the indication of ERM/ILM peeling. Consistently, it has been reported that outer retinal characteristics seem to be vital in predicting visual outcomes after macular surgery [11].

\section{Methods}

2.1. Patient Selections. This was a retrospective medical review (January 2010 to December 2018) of 38 patients who underwent 23-gauge PPVT with ICG-assisted ERM/ILM peeling, as a treatment for idiopathic macular pucker. This study was approved by the Institutional Review Board of the Cheng Hsin General Hospital (CHGH-IRB; Taipei, Taiwan; approval no. (641)107-12) and followed the tenets of the Declaration of Helsinki.

The inclusion criteria of this study include patients with idiopathic ERM together with the performance of 23-gauge PPVT and ICG-assisted EMM/ILM peeling. They were able to return for all regular postpeeling follow-ups. On the other hand, patients were excluded if they had following conditions, namely, presence of ERM secondary to such as trauma, retinal vascular diseases (e.g., diabetic retinopathy, vascular occlusion, and retinal detachment), intraocular inflammation, other diseases that were interfered with the functional results (e.g., high myopia of more than 6 diopters and severe cataract of more than degree 3 nuclear sclerosis/ cortical opacity), and other ocular pathologic features that can affect the central retinal thickness (e.g., diabetic macular edema, glaucoma, age-related macular degeneration, and optic nerve diseases). Finally, patients with previous vitreoretinal surgery were also excluded.

2.2. Preoperative and Postoperative Comprehensive Ophthalmologic Examinations. One of each patient's eyes in the present study underwent pre- and postoperative comprehensive ophthalmologic examinations, which include BCVA measurement (Snellen E; LogMAR), slit-lamp biomicroscopy with a noncontact lens, indirect ophthalmoscopy, color fundus photography (Canon, Tokyo, Japan), metamorphopsia score recording (Amsler grid chart), and SDOCT (Spectralis, Heidelberg Engineering, Heidelberg, Germany). Specifically, visual outcomes of BCVA \pm standard deviation (SE; Snellen E; LogMAR), metamorphopsia score (distorted image: none, 0; mild, 1; moderate, 2; severe, 3), general ophthalmic conditions, outer plexiform layer angle (OPL angle), and CRT (from ILM to RPE) were measured at the baseline and during the follow-up, via usage of SD-OCT. The OPL angle was measured using the On-Screen Protractor Program (Minimum Java Runtime Environment, version 1.7; GNU's Not Unix General Public License, version 3). The OPL angle is calculated from two lines drawn from the OPL at the fovea to the furthermost intersecting point at either side of the OPL. Also, an analyzation included the correlations between postpeeling visual outcomes and various prepeeling parameters, such as CRTs $\geq 500 \mu \mathrm{m}$ vs. $<500 \mu \mathrm{m}$ or OPL angles $\geq 130^{\circ}$ vs. $<130^{\circ}$.

\subsection{Pars Plana Vitrectomy with Indocyanine Green-Assisted} Peeling of ERM and ILM. In all the patients, the 23-gauge PPVT with indocyanine green-assisted ERM/ILM peeling for idiopathic macular pucker was conducted by the same senior vitreoretinal specialist (CHM). ERM/ILM removal was done with intraocular end-gripping forceps following a standard three-port 23-gauge transconjunctival PPVT using the Alcon Constellation Vision System (Alcon Surgical, Fort Worth, Texas, USA). Of note, ILM peeling was carried out simultaneously or immediately after the ERM removal. Then, $0.05 \%$ indocyanine green (ICG; Pulsion Medical systems AG, Munich, Germany) was injected gently over posterior pole, in order stain the ILM for visibility. After the staining was completed, the ICG was suctioned out for a total of $30 \mathrm{~s}$. In this case, the area of ERM/ILM peeling was 2 to 3 disc diameters around the fovea. If the patient's cataract affected the surgery, phacoemulsification with implantation of an intraocular lens was performed prior to the PPVT and membrane peeling treatment. 
2.4. Statistical Analysis. Comparisons between two groups were made using unpaired Student's $t$-tests. All results were shown as means \pm SE. A value of $p<0.05$ was considered significant.

\section{Results}

3.1. Clinical Characteristics of Patients. Among these patients diagnosed with idiopathic macular pucker, 15 were men and 23 were women ranging in age from 43.30 to 86.00 years (mean \pm SD: $65.01 \pm 1.39$ ). The mean postoperative follow-up period was $36.07 \pm 4.62$ months (range from 1.30 to 96.70 months). The period from the $1^{\text {st }}$ diagnosis of idiopathic macular pucker to ERM/ILM peeling is $13.46 \pm 3.50$ months.

3.2. Changes of Prepeeling and Postpeeling Parameters in Metamorphopsia Scores, CRT, and BCVA. The metamorphopsia scores significantly reduced from prepeeling $1.42 \pm 0.16$ to final postpeeling $0.61 \pm 0.08(p<0.001$; Figure 1, top left). CRT also significantly decreased from prepeeling $519.62 \pm 13.41 \mu \mathrm{m}$ to final postpeeling $385.37 \pm 8.97 \mu \mathrm{m} \quad(p<0.001$; Figure 1 , top right $)$. On the other hand, BCVA significantly improved from prepeeling $0.26 \pm 0.03(=0.67 \pm 0.04 \operatorname{LogMAR})$ to final postpeeling $0.67 \pm 0.04 \quad(=0.30 \pm 0.05$ LogMAR; $p<0.001$; Figure 1 , bottom left and right).

3.3. The Correlations among the Prepeeling OPL Angle, Postpeeling BCVA, and Postpeeling Metamorphopsia. In the present study, the OPL angle was defined in the methods and directly calculated (Figure 2). Of novelty to this study, greater prepeeling OPL angle $\left(\geq 130^{\circ}\right)$ was associated with significant postpeeling BCVA improvement $(p<0.001$; $n=14 ; 0.47 \pm 0.06=-0.52 \pm 0.07$ LogMAR) and significant $(p<0.01)$ postpeeling metamorphopsia reduction $(n=14$; $-0.93 \pm 0.20)$. In contrast, a smaller OPL angle $\left(<130^{\circ}\right)$ group showed less postpeeling BCVA improvement $(n=24$; $0.27 \pm 0.05=-0.29 \pm 0.08$ LogMAR) and less postpeeling metamorphopsia reduction $(n=24 ;-0.75 \pm 0.22$; Figure 3$)$.

Furthermore, there were significant differences in pre- to postpeeling changes of BCVA $(p=0.01)$ and LogMAR $(p=$ 0.03 ) between two groups. The multivariate analysis also indicated that the effect of cataract removal (combined cataract operation eye number) and time of ERM presence (period from puckering diagnosis to ERM/ILM peeling) had no significant influence on the comparison of defined postpeeling visual outcome changes between these two OPL angle groups.

\subsection{The Correlations among the Prepeeling CRT, Postpeeling} BCVA, and Postpeeling Metamorphopsia. Smaller $(<500 \mu \mathrm{m})$ prepeeling central foveal thickness was not associated with significant postpeeling BCVA improvement $(n=19$; $0.36 \pm 0.06=-0.39 \pm 0.09)$ and postpeeling metamorphopsia reduction $(n=19 ;-0.89 \pm 0.24)$, as compared to greater central foveal thickness $(\geq 500 \mu \mathrm{m})$ with postpeeling BCVA improvement $\quad(n=19 ; \quad$ BCVA: $\quad 0.33 \pm 0.06=-0.36 \pm 0.08$ LogMAR) and postpeeling metamorphopsia reduction ( $n=19 ;-0.74 \pm 0.18$; Figure 4$)$. Nevertheless, there were no significant intraoperative adverse complications, such as endophthalmitis, retinal tear, and retinal detachment that affected the functional anatomical outcomes after surgery or during follow-up periods.

\section{Discussion}

As mentioned previously, PPVT with ERM/ILM peeling has been advocated as a conventional surgical procedure for treating patients with idiopathic macular pucker (ERM). The combination of indocyanine green-assisted ILM peeling along with ERM removal has been reported to reduce the incidence of recurrence of macular pucker [9, 12]. More importantly, the present study demonstrated that certain prognostic factors, such as CRT thickness and OPL angle, might have correlations with visual outcomes for idiopathic macular pucker patients, who underwent PPVT combined with ERM plus indocyanine green-assisted ILM peeling.

It is acknowledged that BCVA is an important parameter to predict visual recovery after successful ERM/ILM peeling [5, 13-15]. As shown in Figure 1, a significant postpeeling BCVA improvement after surgery was present. Song et al. have indicated that the ganglion cell layer thickness is related to visual improvement after ERM removal [16]. The present investigation explored whether the OPL angle could act as a prognostic factor that predicts the visual function alteration following ERM/ILM peeling for macular pucker patients.

On the other hand, metamorphopsia is one of the most important symptoms in idiopathic macular pucker patients. It has been reported to result from the disarray of photoreceptors induced by tangential traction or contraction of membrane in idiopathic macular pucker patients [17]. The microstructural change was alleviated after successful ERM/ ILM peeling to obtain better postoperative visual outcomes. By doing so, it results in significant BCVA improvement and metamorphopsia reduction [18-20], which is also consistent with a previous study [21]. Bae et al. [22] revealed a fact that the status of postoperative metamorphopsia was dependent on the degree of preoperative metamorphopsia. As indicated by Bae et al. [22], patients with severe preoperative metamorphopsia showed a high level of postoperative residual metamorphopsia. This explains why the present study (Figure 1) also found out that metamorphopsia, though with significant improvements, was still observed partially (score: prepeeling 1.42 to postpeeling 0.61 ) during the mean postoperative follow-up period (36.07 months).

4.1. Prepeeling Greater OPL Angle vs. Smaller OPL Angle Group: Significant Different Final Visual Results. As demonstrated in Figure 3, the present study demonstrated that greater preoperative OPL angle $\left(\geq 130^{\circ}\right)$ was associated with significantly greater BCVA improvement (either Snellen E or LogMAR) and metamorphopsia reduction to a greater extent, when compared with smaller preoperative OPL angle $\left(<130^{\circ}\right)$ that presented with less BCVA improvement and 


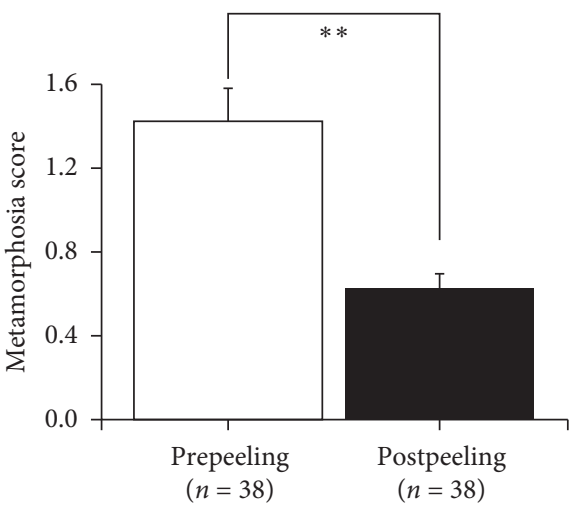

(a)

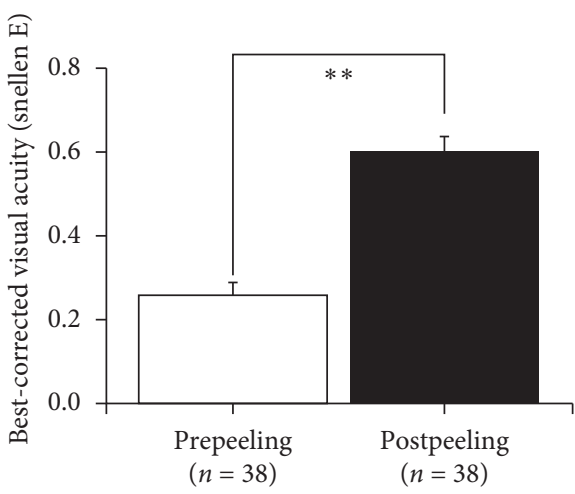

(c)

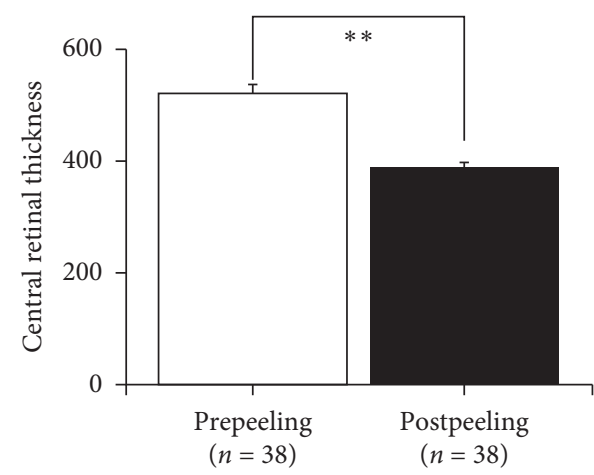

(b)

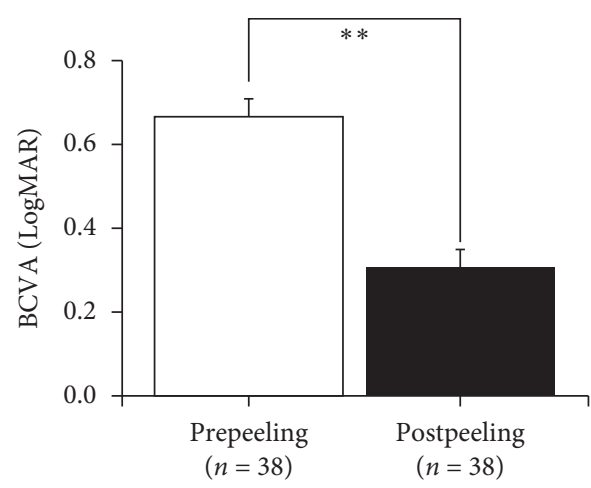

(d)

FiguRE 1: (a) As compared to the baseline (prepeeling), postpeeling metamorphopsia score was significantly improved (reduced score). (b) As compared to the baseline (prepeeling), postpeeling CRT was significantly decreased. (c) and (d) As compared to the baseline (prepeeling), postpeeling BCVA was significantly improved (increased Snellen E and decreased LogMAR), respectively. Abbreviations: CRT, central retinal thickness; BCVA, best-corrected visual acuity; LogMAR: Logarithm of the minimum angle of resolution.
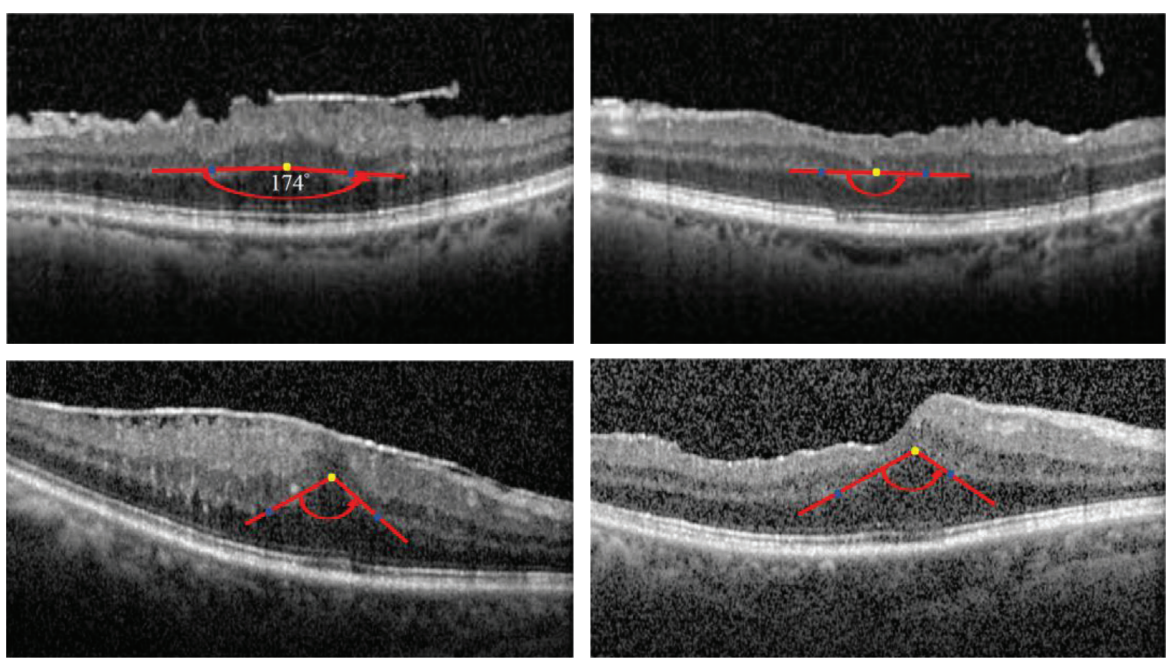

Figure 2: The OPL angle is measured from two lines (red) drawn from the OPL at the fovea (illustrated by yellow dots) to the furthermost intersecting point at either side of the OPL (illustrated by blue dots). (a) Patients' retinas with greater prepeeling OPL angle ( $\left.\geq 130^{\circ}\right)$. (b) Patients' retinas that had smaller prepeeling OPL angle $\left(<130^{\circ}\right)$. (c) Pre-ERM/ILM peeling OCT images (with the OPL angle of $174^{\circ}$ as an example at the top left OCT image). (d) Post-ERM/ILM peeling OCT images. Abbreviations: OPL, outer plexiform layer; OCT, optical coherent tomography; ERM, epiretinal membrane; ILM, internal limiting membrane. 


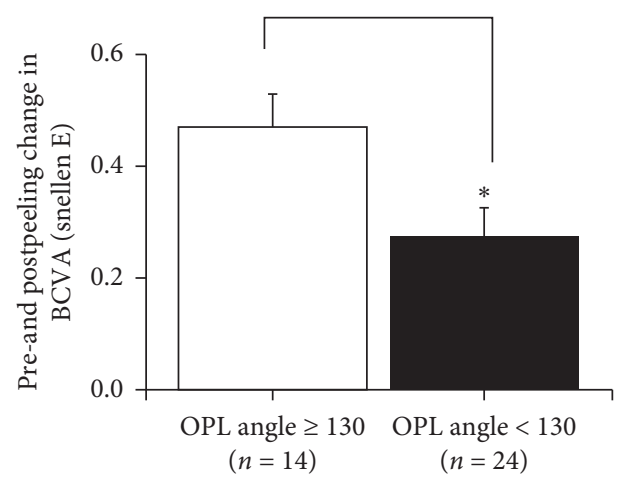

(a)

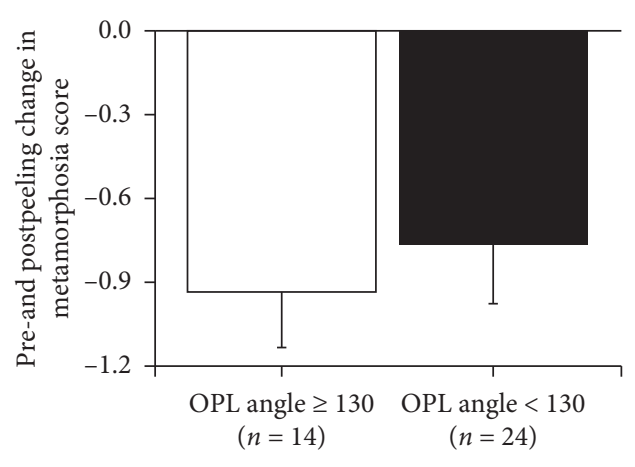

(b)

FIGURE 3: The group of patients' retinas with greater prepeeling OPL angle $\left(\geq 130^{\circ}\right)$ was associated with significant greater BCVA improvement $(n=14 ; 0.47 \pm 0.06=-0.52 \pm 0.07$ LogMAR $)$ and greater metamorphopsia reduction $(n=14 ;-0.93 \pm 0.20)$ as compared to the other group of patients whose retinas had smaller OPL angle $\left(<130^{\circ}\right)$ with less BCVA improvement $(n=24 ; 0.27 \pm 0.05=-0.29 \pm 0.08$ LogMAR) and less metamorphopsia reduction $(n=24 ;-0.75 \pm 0.22)$.

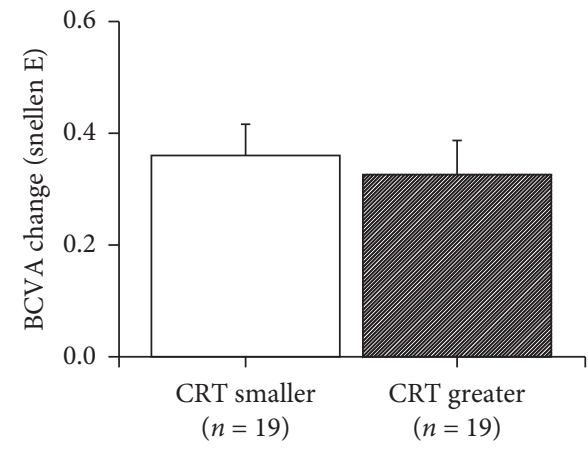

(a)

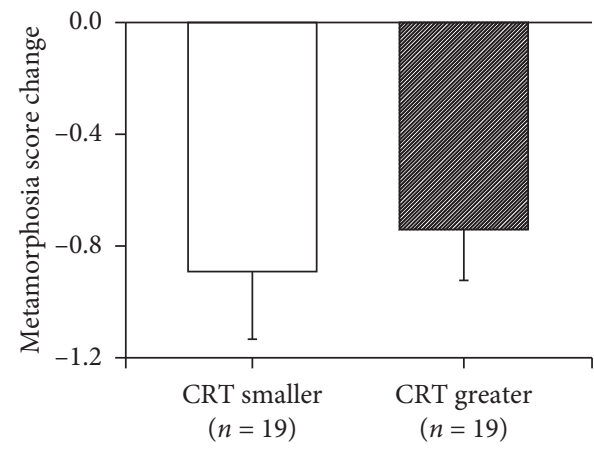

(b)

FIGURE 4: The final postpeeling BCVA improvements ( $n=19 ; 0.36 \pm 0.06=-0.39 \pm 0.09$ LogMAR) and metamorphopsia reductions $(n=19$; $-0.89 \pm 0.24)$ relevant to the group of patients' retinas with the prepeeling less CRT $(<500 \mu \mathrm{m})$ did not significantly differ from those data $(n=19$; BCVA: $0.33 \pm 0.06=-0.36 \pm 0.08$; metamorphopsia score: $-0.74 \pm 0.18)$ relevant to the other group of patients whose retinas had the prepeeling greater CRT $(\geq 500 \mu \mathrm{m})$.

metamorphopsia reduction. These results postulated that the prepeeling OPL angle might be utilized as a novel prognostic factor to predict the postoperative visual outcomes in patients with idiopathic ERM. Whether greater preoperative OPL angle $\left(\geq 130^{\circ}\right)$ indicating less retinal conformation change is linked with significant better postpeeling visual outcomes needs further confirmation. However, the present study has demonstrated that prepeeling OPL angle is a significantly and clinically important factor that could be helpful in predicting postpeeling visual outcomes and justifying the indication of ERM/ILM peeling. As mentioned, it has been indicated that outer retinal features appear to be more important in predicting visual results following macular operation [11].

\subsection{Prepeeling Greater CRT Group vs. Smaller CRT Group: No} Significant Difference in Final Visual Outcomes. To the best of our knowledge, macular pucker significantly affected the retinal thickness and underlying microstructures, when compared with the normal eye. In the present study, the measurement by means of SD-OCT demonstrated that CRT had a significant decrease after PPVT combined with ERM and indocyanine green-assistant ILM peelings, namely, from prepeeling $519.62 \pm 13.41 \mu \mathrm{m}$ to final postpeeling $385.37 \pm 8.97 \mu \mathrm{m}$ (Figure 1). This conforms with previous studies [18-20]. However, the final postoperative BCVA improvement and metamorphopsia reduction relevant to the preoperative greater CRT $(\geq 500 \mu \mathrm{m})$ did not significantly differ from that relevant to the preoperative smaller CRT $(<500 \mu \mathrm{m})$, as shown in Figure 4. Okamoto et al. [23] demonstrated that the degree of metamorphopsia is associated with INL thickness in patients with idiopathic ERM. Not inconsistent with the present results, Bae et al. [22] reported that reduction of postoperative metamorphopsia paralleled with decrease of CRT and improvement of BCVA. Of note, some investigators $[24,25]$ reported CRT was increased after peeling ILM, which might result from mechanical stress on Müller cells induced by peeling manipulation and resulted in swelling, while others held a contrasting view that macular swelling caused by peeling 
ILM might not have an influence on postoperative BCVA [26].

Several studies had advocated that combination of ERM removal with indocyanine green-assisted ILM peeling might lead to unexpected postoperative visual outcomes and even cause a higher incidence of complications $[8,9,27]$. During the follow-up period of the present study, as mentioned in the results, there were no significant adverse complications, namely, endophthalmitis, retinal tear, and retinal detachment. Song et al. [28] demonstrated that poorer prepeeling BCVA more likely achieved a better visual improvement. The researchers of other publications regarding pucker correlated macular conditions such as vitreomacular traction and vitreomacular adhesion [29-31] carried out investigations into predictive visual outcome factors including optical coherence tomography pattern (e.g., vitreomacular adhesion $\leq 1500 \mu \mathrm{m}$ ), shorter symptom duration, and younger age. In the future study, these prognostic factors might also need further investigation.

The present study possesses some limitations. Firstly, data were collected by a retrospective design, which had potential biases on analyzing results. Secondly, a relatively small number of patients were enrolled into this study. Finally, the measurement of metamorphopsia score were performed by the Amsler grid chart. This method was difficult to quantify the exact metamorphopsia score because it required patients to self-describe the degree of image distortion. It might be better that M-charts (Inami CO, Tokyo, Japan) could be used in advanced investigations for easily quantitating the metamorphopsia score by only answering whether the line is straight.

\section{Conclusions}

Indocyanine green-assisted ILM peeling combined with small gauge vitrectomy and ERM peeling is associated with significant visual acuity improvement and metamorphopsia reduction in patients with idiopathic ERM. More importantly, greater prepeeling OPL angles might be able to act as a novel and helpful prognostic factor, in terms of predicting a better final visual outcome in idiopathic macular pucker surgery.

\section{Data Availability}

The data shown and/or analyzed during the present investigation are available from the corresponding author upon a reasonable request.

\section{Disclosure}

This study was presented at the Annual Conference, Taiwan Ophthalmological Society, on November $18^{\text {th }}, 2017$.

\section{Conflicts of Interest}

The authors have no conflicts of interest.

\section{Acknowledgments}

The authors thank Professor Ralph Kirby and Professor Howard Chao for their expertise and kindness in correcting all grammatical and spelling errors of the manuscript.

\section{References}

[1] P. Mitchell, W. Smith, T. Chey, J. J. Wang, and A. Chang, "Prevalence and associations of epiretinal membranes," Ophthalmology, vol. 104, no. 6, pp. 1033-1040, 1997.

[2] R. Machemer, "The surgical removal of epiretinal macular membranes (macular puckers) (author's transl)," Klinische Monatsblatter Augenheilkunde, vol. 173, no. 1, pp. 36-42, 1978.

[3] J. G. Wong, N. Sachdev, P. E. Beaumont, and A. A. Chang, "Visual outcomes following vitrectomy and peeling of epiretinal membrane," Clinical and Experimental Ophthalmology, vol. 33, no. 4, pp. 373-378, 2005.

[4] T. A. Rice, S. De Bustros, R. G. Michels, J. T. Thompson, S. M. Debanne, and D. Y. Rowland, "Prognostic factors in vitrectomy for epiretinal membranes of the macula," Ophthalmology, vol. 93, no. 5, pp. 602-610, 1986.

[5] M. Inoue, S. Morita, Y. Watanabe et al., "Preoperative inner segment/outer segment junction in spectral-domain optical coherence tomography as a prognostic factor in epiretinal membrane surgery," Retina, vol. 31, no. 7, pp. 1366-1372, 2011.

[6] M. Shimozono, A. Oishi, M. Hata et al., "The significance of cone outer segment tips as a prognostic factor in epiretinal membrane surgery," American Journal of Ophthalmology, vol. 153, no. 4, pp. 698-704, 2012.

[7] Y. Itoh, M. Inoue, T. Rii, K. Hirota, and A. Hirakata, "Correlation between foveal cone outer segment tips line and visual recovery after epiretinal membrane surgery," Investigative Opthalmology and Visual Science, vol. 54, no. 12, pp. 73027308, 2013.

[8] R. Grewing and U. Mester, "Results of surgery for epiretinal membranes and their recurrences," British Journal of Ophthalmology, vol. 80, no. 4, pp. 323-326, 1996.

[9] A. K. Kwok, T. Y. Lai, and K. S. Yuen, "Epiretinal membrane surgery with or without internal limiting membrane peeling," Clinical and Experimental Ophthalmology, vol. 33, no. 4, pp. 379-385, 2005.

[10] R. Schadlu, S. Tehrani, G. K. Shah, and A. G. Prasad, "Longterm follow-up results of ilm peeling during vitrectomy surgery for premacular fibrosis," Retina, vol. 28, no. 6, pp. $853-857,2008$.

[11] N. Villate, J. E. Lee, A. Venkatraman, and W. E. Smiddy, "Photoreceptor layer features in eyes with closed macular holes: optical coherence tomography findings and correlation with visual outcomes," American Journal of Ophthalmology, vol. 139, no. 2, pp. 280-289, 2005.

[12] D. W. Park, P. U. Dugel, J. Garda et al., "Macular pucker removal with and without internal limiting membrane peeling: pilot study," Ophthalmology, vol. 110, no. 1, pp. 62-64, 2003.

[13] R. Asaria, L. Garnham, Z. J. Gregor, and J. J. Sloper, “A prospective study of binocular visual function before and after successful surgery to remove a unilateral epiretinal membrane," Ophthalmology, vol. 115, no. 11, pp. 1930-1937, 2008.

[14] A. Shiono, J. Kogo, G. Klose et al., "Photoreceptor outer segment length: a prognostic factor for idiopathic epiretinal 
membrane surgery," Ophthalmology, vol. 120, no. 4, pp. 788-794, 2013.

[15] H. Kunikata, T. Abe, J. Jiro Kinukawa, and K. Nishida, "Preoperative factors predictive of postoperative decimal visual acuity $\geq 1.0$ following surgical treatment for idiopathic epiretinal membrane," Clinical Ophthalmology, vol. 5, pp. 147-154, 2011.

[16] S. J. Song, M. Y. Lee, and W. E. Smiddy, "Ganglion cell layer thickness and visual improvement after epiretinal membrane surgery," Retina, vol. 36, no. 2, pp. 305-310, 2016.

[17] R. G. Michels, "A clinical and histopathologic study of epiretinal membranes affecting the macula and removed by vitreous surgery," Transactions of the American Ophthalmological Society, vol. 80, pp. 580-656, 1982.

[18] T. Kinoshita, H. Imaizumi, U. Okushiba, H. Miyamoto, T. Ogino, and Y. Mitamura, "Time course of changes in metamorphopsia, visual acuity, and OCT parameters after successful epiretinal membrane surgery," Investigative Opthalmology \& Visual Science, vol. 53, no. 7, pp. 3592-3597, 2012.

[19] J. H. Kim, S. W. Kang, M. G. Kong, and H. S. Ha, “Assessment of retinal layers and visual rehabilitation after epiretinal membrane removal," Graefe's Archive for Clinical and Experimental Ophthalmology, vol. 251, no. 4, pp. 1055-1064, 2013.

[20] F. Okamoto, Y. Sugiura, Y. Okamoto, T. Hiraoka, and T. Oshika, "Inner nuclear layer thickness as a prognostic factor for metamorphopsia after epiretinal membrane surgery," Retina, vol. 35, no. 10, pp. 2107-2114, 2015.

[21] F. Okamoto, Y. Okamoto, T. Hiraoka, and T. Oshika, "Effect of vitrectomy for epiretinal membrane on visual function and vision-related quality of life," American Journal of Ophthalmology, vol. 147, no. 5, pp. 869-874.E1, 2009.

[22] S. H. Bae, D. Kim, T. K. Park, J. R. Han, H. Kim, and W. Nam, "Preferential hyperacuity perimeter and prognostic factors for metamorphopsia after idiopathic epiretinal membrane surgery," American Journal of Ophthalmology, vol. 155, no. 1, pp. 109-117.E1, 2013.

[23] F. Okamoto, Y. Sugiura, Y. Okamoto, T. Hiraoka, and T. Oshika, "Associations between metamorphopsia and foveal microstructure in patients with epiretinal membrane," Investigative Opthalmology and Visual Science, vol. 53, no. 11, pp. 6770-6775, 2012.

[24] G. Ripandelli, F. Scarinci, P. Piaggi et al., "Macular pucker: to peel or not to peel the internal limiting membrane? A microperimetric response," Retina, vol. 35, no. 3, pp. 498-507, 2015.

[25] J. W. Lee and I. T. Kim, "Outcomes of idiopathic macular epiretinal membrane removal with and without internal limiting membrane peeling: a comparative study," Japanese Journal of Ophthalmology, vol. 54, no. 2, pp. 129-134, 2010.

[26] A. Clark, N. Balducci, F. Pichi et al., "Swelling of the arcuate nerve fiber layer after internal limiting membrane peeling," Retina, vol. 32, no. 8, pp. 1608-1613, 2012.

[27] M. Maia, E. Margalit, R. Lakhanpal et al., "Effects of intravitreal indocyanine green injection in rabbits," Retina, vol. 24, no. 1, pp. 69-79, 2004.

[28] S. J. Song, A. E. Kuriyan, and W. E. Smiddy, "Results and prognostic factors for visual improvement after pars plana vitrectomy for idiopathic epiretinal membrane," Retina, vol. 35, no. 5, pp. 866-872, 2015.

[29] C. Y. Tsai, Y. T. Hsieh, T. T. Lai, and C. M. Yang, "Idiopathic macular holes and direction of vitreomacular traction: structural changes and surgical outcomes," Eye, vol. 31, no. 12, pp. 1689-1696, 2017.

[30] D. Y. Liao, J. H. Liu, Y. P. Zheng, H. W. Shiu, J. M. Wang, and H. M. Chao, "OCT proves that vitreomacular adhesion is significantly more likely to develop vision-threatening retinal complications than vitreomacular separation," BMC Ophthalmology, vol. 20, no. 1, p. 163, 2020.

[31] C. S. Yang, M. H. Hsieh, Y. F. Chang, C. Y. Wang, and S. J. Chen, "Predictive factors of visual outcome for vitreomacular traction syndrome after vitrectomy," Retina, vol. 38, no. 8 , pp. $1533-1540,2018$. 